Projets

de paysage

\section{Projets de paysage}

Revue scientifique sur la conception et l'aménagement de l'espace

$5 \mid 2011$

Le jardin et ses rapports à l'art. Encadrer, décadrer, recadrer

\title{
Gaëlle Aggéri, Inventer les villes-natures de demain... Gestion différenciée, gestion durable des espaces verts
}

Note de lecture

Gaëlle Aggéri, Inventer les villes-natures de demain... Gestion différenciée, gestion durable des espaces verts - Reading report

\section{Audrey Marco}

\section{OpenEdition}

Journals

\section{Édition électronique}

URL : https://journals.openedition.org/paysage/22315

DOI : $10.4000 /$ paysage.22315

ISSN : 1969-6124

\section{Éditeur :}

École nationale supérieure du paysage de Versailles-Marseille, Institut national des sciences appliquées Centre Val de Loire - École de la nature et du paysage, École nationale supérieure d'architecture et de paysage de Bordeaux, École nationale supérieure d'architecture et de paysage de Lille, Agrocampus Angers

\section{Référence électronique}

Audrey Marco, « Gaëlle Aggéri, Inventer les villes-natures de demain... Gestion différenciée, gestion durable des espaces verts », Projets de paysage [En ligne], 5 | 2011, mis en ligne le 19 janvier 2011, consulté le 16 octobre 2021. URL : http://journals.openedition.org/paysage/22315 ; DOI : https://doi.org/ 10.4000/paysage. 22315

Ce document a été généré automatiquement le 16 octobre 2021

\section{(c) $)(9)$}

La revue Projets de paysage est mise à disposition selon les termes de la Licence Creative Commons Attribution - Pas d'Utilisation Commerciale - Pas de Modification 4.0 International. 


\section{Gaëlle Aggéri, Inventer les villes-} natures de demain... Gestion différenciée, gestion durable des espaces

\section{verts}

Note de lecture

Gaëlle Aggéri, Inventer les villes-natures de demain... Gestion différenciée, gestion durable des espaces verts - Reading report

\section{Audrey Marco}

\section{RÉFÉRENCE}

Gaëlle Aggéri, Inventer les villes-natures de demain... Gestion différenciée, gestion durable des espaces verts, Dijon, Educagri éditions, 2010, 199 p.

\section{L'auteur}

Gaëlle Aggéri, ingénieure paysagiste, a été quinze ans responsable du bureau d'études Paysage au service espaces verts de la ville de Montpellier, puis sept ans responsable de formation au Centre national de la fonction publique territoriale. Elle est actuellement chercheur associée au laboratoire de l'École nationale supérieure du paysage de Versailles (Larep). 


\section{L'auteur de la préface}

2 Pierre Donadieu, géographe-agronome, est professeur à l'École nationale supérieure du paysage de Versailles-Marseille et chercheur à l'Institut national de la recherche agronomique au sein de l'UMR SAD-APT de Paris-Grignon.

\section{L'auteur de la postface}

3 Bernadette Lizet, ethnologue, est directrice de recherches au Muséum national d'histoire naturelle de Paris au sein de l'UMR 7206.

\section{Structure et contenu du livre}

4 Les derniers changements survenus en matière de gestion et de conception des espaces verts urbains n'avaient pas encore été abordés par la production scientifique alors qu'ils participent aujourd'hui à une révolution des valeurs culturelles et des pratiques culturales dans l'histoire des aménagements publics urbains. L'ouvrage conçu par Gaëlle Aggéri est un livre que l'on attendait donc depuis longtemps! Il permet de cristalliser l'aventure de la "gestion différenciée ", nouveau mode de gestion qui s'est peu à peu imposé ces dernières décennies dans les services espaces verts et les collectivités territoriales. Il pointe également, sous l'impulsion de la mouvance du développement durable, les nouvelles façons de penser ou d' «inventer les villesnatures de demain... ».

Ce livre se structure en quatre parties avec quelques pages en guise d'introduction et de conclusion.

Dans la première partie, l'auteur propose de retracer au cours du temps la succession des différents évènements relatifs à l'origine et à la diffusion des idées sur la nature urbaine en Europe, et aux répercussions qu'ils ont pu avoir sur l'ancrage du mouvement de la gestion différenciée sur le territoire français. Ce flash-back permet de mettre en exergue les précurseurs et les catalyseurs de ce mouvement qui ont conduit à une nouvelle façon de penser et de gérer les espaces verts en ville. En adoptant une démarche rétrospective, l'auteur nous resitue tout d'abord dans le contexte des années 1970 face à la décadence de la gestion horticole. Sont ainsi présentés les divers aspects de cette gestion intensive qui ne font plus l'unanimité auprès du monde professionnel notamment la simplification dans la conception des espaces verts, la valorisation d'espaces verts fonctionnels, la standardisation des techniques horticoles intensives, chimiques et mécanisées développées pour l'entretien. Dans le chapitre suivant, l'auteur élargit son regard à l'international, et identifie en Europe du Nord les modèles de gestion plus proches de la nature qui ont pu influencer à cette époque les directeurs des services espaces verts français. Ce chapitre se poursuit logiquement sur les différents acteurs et évènements qui ont participé à la diffusion des principes de gestion écologique en France dans les années 1980-1990. Il est ponctué de témoignages de divers acteurs d'espaces publics qui révèlent avec émotion la ténacité dont ils ont fait preuve, d'une part, pour rompre avec leurs savoirs et savoir-faire ancestraux, et d'autre part, pour promouvoir la gestion différenciée auprès des collectivités territoriales et des élus. Ces efforts, non sans peine, ont permis quelques années plus 
tard d'asseoir dans les services d'espaces verts le socle commun de la gestion différenciée et de le diffuser grâce à différents soutiens politiques, techniques, artistiques, scientifiques et urbanistiques.

$7 \mathrm{Au}$ cours de la deuxième partie, l'auteur s'attache au propre de la "gestion différenciée » en commençant par définir ce nouveau mode de gestion au regard des autres types de gestion existants, ainsi que, le vocable et les objectifs qui lui sont associés. La gestion différenciée se présente alors comme une nouvelle alternative à la gestion horticole, privilégiant la diversification des modes d'entretien au regard de la spécificité d'usage, esthétique et écologique de chaque type d'espace vert. Dans le chapitre qui suit, l'auteur nous montre comment cette nouvelle démarche d'entretien s'est inscrite techniquement au sein des services espaces verts à travers la mise en place d'une méthodologie précise de qualification des espaces et de la définition des modalités d'entretien. L'auteur prend alors le parti pris d'illustrer concrètement à partir d'expériences de politiques urbaines les différentes typologies d'espaces verts et objectifs d'entretien qui ont pu être définis. La diversité des récits et des documents présentés (codes, guides et référentiels) montrent comment ces collectivités ont su se réapproprier leur territoire et redéfinir une stratégie d'intervention sur leurs espaces verts à travers l'espace et le temps. Ce chapitre se veut être la traduction d'un travail de terrain. Au-delà de la dimension technique, l'auteur souligne les nouveaux engagements qui accompagnent l'adoption de la gestion différenciée en matière de la préservation des ressources naturelles et donc du positionnement des collectivités au regard de la gestion durable de leur territoire. Cette partie s'achève sur les nouvelles missions qui attendent les élus qui s'engagent dans cette démarche car si un grand nombre de gestionnaires d'espaces verts ont su s'adapter à ce nouveau mode de pensée, nombre de jardiniers et citoyens ne sont pas encore sensibilisés à ces nouvelles pratiques de gestion de leur territoire.

8 Dans la troisième partie, l'auteur a souhaité faire une analyse des référentiels de nature qui accompagnent actuellement la conception des espaces verts urbains porteurs d'une gestion différenciée. Mais avant de les reprendre plus en détail, l'auteur consacre un premier chapitre sur l'évolution de la demande sociale en matière de nature et de paysage dans les espaces publics depuis les années 1970 afin de décrypter les attentes et les usages de nature des citadins dans ces espaces. Ce décryptage est réalisé grâce à quelques enquêtes conduites par les services espaces verts eux-mêmes qui souhaitaient faire réagir les citadins sur les nouvelles productions paysagères conçues à l'époque. Les chapitres qui suivent montrent alors comment les "villes-natures" de demain peuvent répondre à leurs attentes grâce à une analyse par milieu des formes et des ambiances paysagères synchrones à la diffusion de la gestion différenciée. L'auteur a choisi de faire une classification de l'offre de nature selon les trois types de natures historiquement reconnues - la nature horticole, la nature champêtre, la nature sauvage. Le référentiel horticole est rapidement exposé par l'auteur qui s'attarde plus longuement sur les intentions des concepteurs et les référents en termes d'inspiration et d'imaginaire sur la nature champêtre et sauvage. Des fiches projets illustrent les matières végétales, les mobiliers urbains, les animations... qui traduisent ces différentes conceptions de nature.

9 «Au-delà de la gestion différenciée : quelles nouvelles politiques durables de la nature en ville? ", c'est ainsi que s'intitule la dernière partie de cet ouvrage. L'auteur nous invite ici à réfléchir plus largement sur les incidences de la gestion différenciée et de 
l'émergence du concept de développement durable dans la façon de concevoir les projets urbains. Cela nécessite d'opérer, d'une part, un changement d'échelle, il ne s'agit plus de rester à l'échelle du service espace vert mais bien à l'ensemble des services intervenant sur le territoire, et d'autre part, une "synergie» entre les partenaires du projet urbain afin de mener une réflexion collective sur les différents aspects qu'impose la notion de durabilité. Pour conduire sa réflexion autour de la planification des territoires urbains, de la prise en compte de la préservation des ressources naturelles et de la relation homme/nature dans les villes de demain, l'auteur s'appuie sur des expériences paysagères durables de la période de l'après-gestion différenciée, explorant à la fois le champ social, économique, et environnemental. Des fiches projets et des illustrations de sites conçus transversalement selon une logique de développement durable à toutes les échelles viennent alimenter le débat. Cette dernière partie interpelle donc tous les acteurs des villes sur la nécessité d'une profonde remise en question des méthodes de travail et de conception existantes afin de mieux répondre aux vocations des " villes-natures de demain ».

En conclusion, cet ouvrage se présente comme une contribution particulièrement intéressante non seulement pour dresser un état synthétique des connaissances actuelles sur la gestion différenciée, mais aussi pour le développement de recherches futures, interdisciplinaires, sur la thématique de la ville durable.

11 Cet ouvrage se veut optimiste et visionnaire. L'auteur tient à mettre un point d'honneur sur l'appropriation de la démarche de la gestion différenciée et de son adaptation au patrimoine vert auprès de l'ensemble des moyennes et grandes villes françaises, ainsi que sur le développement actuel de démarches de politiques urbaines plus globales répondant aux nouvelles vocations des « villes-natures ». Cependant, il est important de souligner qu'il reste encore de nombreuses disparités entre les villes et qu'un grand nombre de communes, de personnels, d'élus et de gestionnaires restent encore réticents face à cette nouvelle démarche. Même si l'auteur n'a pas caché les difficultés qu'ont eues les collectivités pour engager une nouvelle démarche de gestion différenciée dans les services espaces verts, il aurait été intéressant de proposer un volet sur les craintes et difficultés encore existantes aux communes qui ont encore du mal à opérer ce changement de pratique. Ce livre sera peut-être un moyen de les convaincre car un travail important a été conduit dans cet ouvrage pour faire remonter l'expérience du terrain. Il présente en effet un très grand nombre de témoignages, d'exemples de projets et de réalisations, de politiques urbaines, ainsi que d'excellentes photographies qui rendent extrêmement vivant cet ouvrage. Ce livre s'adresse donc à un large public, aux personnels des collectivités territoriales, aux gestionnaires d'espaces publics, aux enseignants, aux chercheurs, aux formateurs mais aussi aux citadins qui s'interrogent sur leur ville. 
INDEX

Keywords : local authorities, urban development plan, differentiated management, biodiversity, sustainable development

Mots-clés : collectivités territoriales, projet de territoire, gestion différenciée, biodiversité, développement durable

\section{AUTEUR}

AUDREY MARCO

Audrey Marco est maître de conférence en écologie végétale à l'École nationale supérieure du paysage - site de Marseille - et chercheur au sein du Laboratoire de recherche sur le paysage de Versailles (Larep).

a.marco[at]ecole-paysage[dot]fr 